\title{
Caracterização citogenética em Schlumbergera truncata (Haworth) Moran e Schlumbergera $\times$ buckleyi (T. Moore) Tjaden (Cactaceae)
}

\author{
Flavia Aparecida Ortolani ${ }^{1,2}$, Márcia Fiorese Mataqueiro ${ }^{1}$ e José Roberto Moro ${ }^{1}$
}

Recebido em 8/05/2006. Aceito em 23/10/2006

\begin{abstract}
RESUMO - (Caracterização citogenética em Schlumbergera truncata (Haworth) Moran e Schlumbergera $\times$ buckleyi (T. Moore) Tjaden (Cactaceae)). O número cromossômico diplóide de Schlumbergera truncata e Schlumbergera x buckleyi, de indivíduos com diferentes tipos de coloração de pétalas, foi determinado usando-se pontas de raízes. A utilização de 8-hidroxiquinoleína $0,003 \mathrm{M}$ à $36{ }^{\circ} \mathrm{C}$ por 3 horas possibilitou melhor separação cromossômica. Técnica de bandeamento C e de coloração Giemsa permitiram o estudo cariológico dessas espécies. O híbrido Schlumbergera $\times$ buckleyi (rósea) apresenta $2 \mathrm{n}=22$ cromossomos com fórmula cariotípica $16 \mathrm{M}+6 \mathrm{SM}$. Schlumbergera truncata, apresentando pétalas nas cores vermelha, branca e pink, possui $2 \mathrm{n}=22$ cromossomos, formulação cariotípica idêntica à de Schlumbergera $\times$ buckleyi, enquanto a planta com flores de coloração amarelada mostrou $2 \mathrm{n}=34$ cromossomos. A classificação cromossômica foi baseada no índice centromérico. Nas plantas que apresentam coloração vermelha, branca, pink e rósea nas pétalas, o melhor período de obtenção de metáfases corresponde ao período de florescimento. Schlumbergera truncata com flores amareladas apresenta dois picos anuais de divisão mitótica. Esses resultados dão suporte à um melhor entendimento da biologia no gênero Schlumbergera e auxiliam na classificação taxonômica nos casos onde apenas as características fenotípicas não são suficientemente confiáveis para a classificação das plantas no mesmo táxon.
\end{abstract}

Palavras-chave: Cactaceae, Cariótipo, Número cromossômico, Banda-C

ABSTRACT - (Cytogenetic characterization of Schlumbergera truncata (Haworth) Moran and Schlumbergera $\times$ buckleyi $($ T. Moore) Tjaden (Cactaceae)). The diploid chromosome number of Schlumbergera truncata and Schlumbergera $\times$ buckleyi, in individuals with different types of petal color, was determined using root tips. The use of 8-hydroxyquinolein $0.003 \mathrm{M}$ at $36^{\circ} \mathrm{C}$ provided better chromosome separation. C-banding technique and Giemsa coloration allowed the karyological study of these species. Schlumbergera $\times$ buckleyi hybrid (light pink) species has $2 \mathrm{n}=22$ chromosomes with karyotype formula $16 \mathrm{M}+6 \mathrm{SM}$. Schlumbergera truncata with red, white, and pink petals and $2 \mathrm{n}=22$ chromosomes has karyotype formula identical to Schlumbergera $\times$ buckleyi, while the plant with yellowish flowers has $2 \mathrm{n}=34$ chromosomes. Chromosome classification was based on the centromeric index. In plants with white, red, pink and light pink petal color, the best time to obtain metaphases is during flowering. Schlumbergera truncata with yellowish flowers has two annual peaks of mitotic division. These results give us a better understanding of the biology of the genus Schlumbergera and aid in taxonomic classification where phenotypic characteristics alone are not reliable enough to classify plants of the same taxon.

Key words: Cactaceae, karyotype, chromosome numbers, C-banding

\section{Introdução}

O gênero Schlumbergera Lemaire (Cactaceae, subtribo Rhipsalidinae) é constituído por seis espécies de arbustos epífitas (Barthlott \& Taylor 1995). Duas dessas espécies, S. truncata (Haworth) Moran e S. russelliana (Hooker) Britton \& Rose são endêmicas do Estado do Rio de Janeiro, Brasil. O híbrido mais importante, do ponto de vista comercial, é denominado $S . \times$ buckleyi (T. Moore) Tjaden, obtido através do cruzamento de $S$. truncata $\times S$. russelliana.
Por muitos anos, os aspectos de biologia molecular (O’Leary \& Boyle 1998) e de biologia reprodutiva (Boyle et al. 1995; Boyle 1997) atraíram a atenção de vários pesquisadores, pois permitem esclarecer, em muitos casos, os fundamentos citológicos e genéticos da variabilidade e sua evolução (Martinez 1976).

Stockwell (1935) tornou-se um dos pioneiros no estudo citogenético de Cactaceae ao analisar 45 espécies. O autor determinou o número cromossômico de $S$. russelliana como sendo $2 \mathrm{n}=22$ cromossomos e encontrou, em algumas espécies de opuntias, níveis variáveis de ploidia $(2 n=44$ e $2 n=66)$. Katagiri (1953),

1 Universidade Estadual Paulista (UNESP/FCAV - Jaboticabal), Laboratório de Citogenética, Departamento de Biologia Aplicada à Agropecuária, Via de Acesso Prof. Paulo Donato Castellane s/n, 14884-900 Jaboticabal, SP, Brasil

2 Autor para correspondência: fortol@ig.com.br 
Remski (1954), Weedin \& Powell (1978) e Ross (1981) relatam que o número básico haplóide, em cactos, é $\mathrm{n}=\mathrm{x}=11$, podendo haver vários níveis de ploidias em alguns gêneros de cactáceas. No entanto, artigos recentes relacionados à citogenética de cactos são praticamente inexistentes. Informações cariotípicas sobre as cactáceas geralmente são restritas à contagem do número de cromossomos (Weedin \& Powell 1978; Ross 1981; Parks \& Boyle 2003). Muitos dos estudos relacionados à esta família direcionam-se às características de morfologia externa (Katagiri 1953), germinação de pólen e desenvolvimento da semente (Boyle et al. 1995), auto-incompatibilidade (Boyle 1997; O'Leary \& Boyle 1998) e importância econômica.

As análises cariotípicas constituem um procedimento de grande utilidade para a diferenciação de certas categorias taxonômicas próximas, particularmente nos casos onde as características fenotípicas não são suficientes para uma separação confiável em táxons distintos. O presente trabalho teve por objetivos caracterizar citogeneticamente a espécie $S$. truncata e o híbrido $S$. × buckleyi, determinar os melhores períodos anuais para obtenção de metáfases nestas duas espécies e realizar um estudo convencional dos cariótipos a fim de diagnosticar características diferenciais, com o auxílio da técnica de bandeamento $\mathrm{C}$.

\section{Material e métodos}

As estacas analisadas foram coletadas de várias plantas diferentes, no município de Guariba (SP), Brasil. Para a cariotipagem, foram avaliadas plantas com cinco diferentes cores: branca, vermelha, pink e amarelada, para a espécie $S$. truncata e rósea, para o híbrido $S$. $\times$ buckleyi.

O enraizamento foi feito em Sphagnum. Raízes com 2,0 cm de comprimento foram coletadas e tratadas com 8 - hidroxiquinoleína $0,003 \mathrm{M}$ por três horas à $36{ }^{\circ} \mathrm{C}$. Em seguida, foram fixadas em solução Carnoy (3 metanol : 1 ácido acético glacial) e mantidas em geladeira por 48 horas. As raízes passaram por três lavagens seguidas, em água destilada, com duração de cinco minutos cada. Posteriormente, foram hidrolisadas em $\mathrm{HCl} 1 \mathrm{~N}$ à $60{ }^{\circ} \mathrm{C}$, por doze minutos e maceradas em ácido acético $45 \%$.

$\mathrm{O}$ bandeamento $\mathrm{C}$ foi realizado de acordo com a metodologia descrita por Guerra \& Souza (2002).

A observação do material foi realizada em microscópio ZEISS com aumento de até 1.000x. Para a contagem dos cromossomos e a cariologia foram analisadas 10 metáfases para cada espécime. Esses procedimentos foram auxiliados pelo sistema de imagem Ikaros (Metasystems). A biometria cromossômica foi efetuada com o programa KS-300, versão 2.02 da Kontron Elektronik, utilizando-se 15 metáfases de cada grupo. Os comprimentos cromossômicos médios e seus respectivos desvios-padrão foram obtidos no programa Excel. Os cromossomos foram classificados de acordo com o índice centromérico seguindo o método proposto por Levan et al. (1964), IC $=\mathrm{BC} \times 100 / \mathrm{T}$, sendo: $\mathrm{IC}=$ índice centromérico, $\mathrm{BC}=$ comprimento do braço curto; $\mathrm{T}=$ comprimento cromossômico total.

\section{Resultados e discussão}

Na espécie $S$. truncata todas as metáfases analisadas, para as variedades com flor de cor branca (Fig. 1), vermelha (Fig. 2) e pink (Fig. 3), apresentaram $2 \mathrm{n}=22$ cromossomos com fórmula cariotípica $16 \mathrm{M}+$ 6SM (Tab. 1). O híbrido S. $\times$ buckleyi apresentou idêntico resultado (Fig. 4). Já a planta $S$. truncata com pétalas de coloração amarelada possui $2 \mathrm{n}=34$ cromossomos (Fig. 5). Na literatura, vários estudos citogenéticos demonstram que o número cromossômico
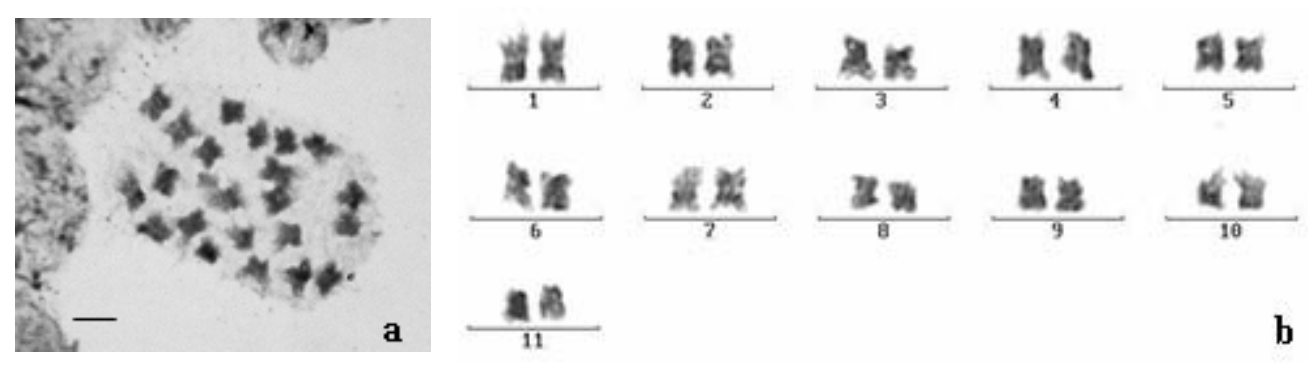

b

Figura 1. a. Metáfase mitótica de S.truncata contendo flores de pigmentação branca. b. Cariótipo mitótico $(2 \mathrm{n}=22$ cromossomos). Barra $=2,5 \mu \mathrm{m}$. 

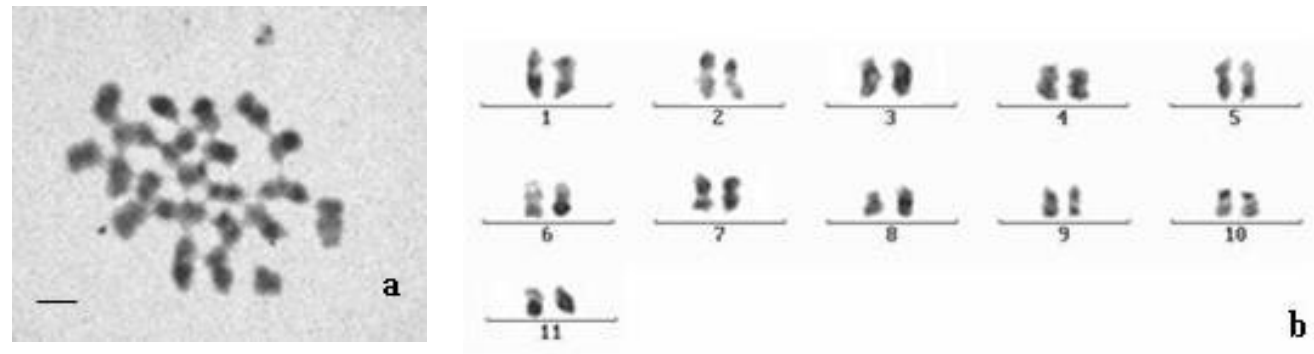

Figura 2. a. Metáfase mitótica de S.truncata contendo flores de pigmentação vermelha. b. Cariótipo mitótico $(2 \mathrm{n}=22$ cromossomos). Barra $=2,5 \mu \mathrm{m}$.
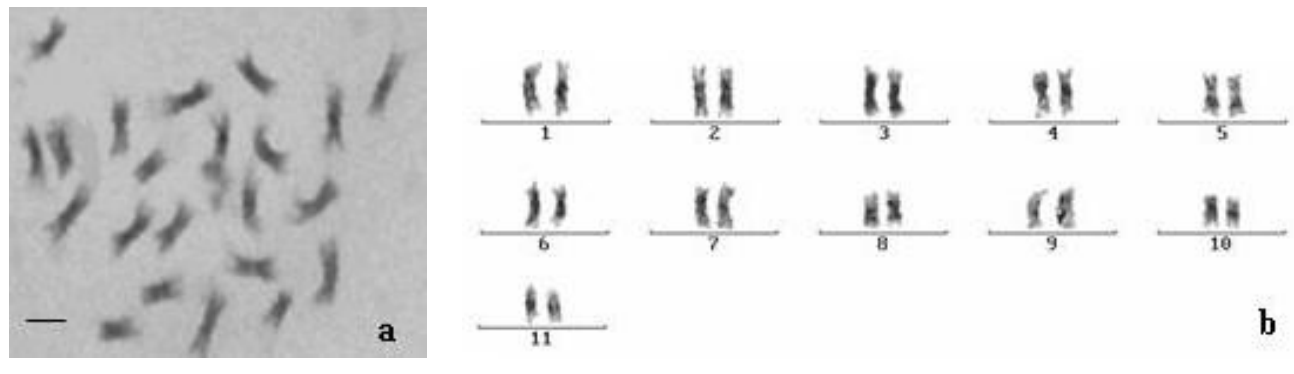

Figura 3. a. Metáfase mitótica de S. truncata contendo flores de pigmentação pink. b. Cariótipo mitótico $(2 \mathrm{n}=22$ cromossomos). Barra $=2,5 \mu \mathrm{m}$.

Tabela 1. Classificação cromossômica da espécie S. truncata e do híbrido $S . \times$ buckleyi (Fórmula cariotípica: 16M + 6SM).

\begin{tabular}{|c|c|c|c|c|c|c|c|c|c|c|c|c|c|}
\hline \multirow[b]{2}{*}{ Par cromossômico } & \multicolumn{3}{|c|}{$\begin{array}{l}\text { S. truncata } \\
\text { branca }\end{array}$} & \multicolumn{3}{|c|}{$\begin{array}{l}\text { S. truncata } \\
\text { vermelha }\end{array}$} & \multicolumn{3}{|c|}{$\begin{array}{l}\text { S. truncata } \\
\text { pink }\end{array}$} & \multicolumn{3}{|c|}{$\begin{array}{c}\text { S. } \times \text { buckleyi } \\
\text { rósea }\end{array}$} & \multirow[b]{2}{*}{ Classificação } \\
\hline & $\mathrm{CB}$ & $\mathrm{T}$ & IC & $\mathrm{CB}$ & $\mathrm{T}$ & IC & $\mathrm{CB}$ & $\mathrm{T}$ & IC & $\mathrm{CB}$ & $\mathrm{T}$ & IC & \\
\hline \multirow[t]{2}{*}{1} & 1,34 & 3,58 & 37,43 & 1,26 & 3,37 & 37,39 & 1,25 & 3,37 & 37,09 & 1,12 & 3,37 & 33,23 & SM \\
\hline & 1,23 & 3,58 & 34,35 & 1,26 & 3,37 & 37,39 & 1,25 & 3,37 & 37,09 & 1,12 & 3,33 & 33,63 & $\mathrm{SM}$ \\
\hline \multirow[t]{2}{*}{2} & 1,23 & 3,47 & 35,45 & 1,10 & 3,07 & 37,16 & 1,26 & 3,37 & 37,38 & 1,23 & 3,27 & 37,30 & SM \\
\hline & 1,23 & 3,47 & 35,45 & 1,13 & 2,96 & 37,16 & 1,26 & 3,37 & 37,38 & 1,23 & 3,27 & 37,30 & $\mathrm{SM}$ \\
\hline \multirow[t]{2}{*}{3} & 1,43 & 3,07 & 46,58 & 1,23 & 2,66 & 46,24 & 1,24 & 3,27 & 38,39 & 1,17 & 3,07 & 38,11 & M \\
\hline & 1,43 & 3,07 & 46,58 & 1,23 & 2,66 & 46,24 & 1,24 & 3,23 & 37,92 & 1,17 & 3,07 & 38,11 & M \\
\hline \multirow[t]{2}{*}{4} & 1,33 & 3,07 & 43,32 & 1,13 & 2,66 & 42,48 & 1,33 & 3,17 & 41,96 & 1,12 & 2,96 & 37,78 & M \\
\hline & 1,33 & 3,07 & 43,32 & 1,12 & 2,55 & 43,92 & 1,33 & 3,17 & 41,96 & 1,12 & 2,96 & 37,78 & M \\
\hline \multirow[t]{2}{*}{5} & 1,33 & 2,96 & 44,93 & 1,12 & 2,45 & 45,71 & 1,23 & 3,07 & 40,07 & 1,23 & 2,96 & 41,55 & M \\
\hline & 1,33 & 2,96 & 44,93 & 1,12 & 2,35 & 47,66 & 1,23 & 3,07 & 40,07 & 1,23 & 2,96 & 41,55 & M \\
\hline \multirow[t]{2}{*}{6} & 1,12 & 2,66 & 42,11 & 1,12 & 2,35 & 47,66 & 1,13 & 2,86 & 39,51 & 1,23 & 2,86 & 43,01 & M \\
\hline & 1,12 & 2,66 & 42,11 & 1,12 & 2,25 & 49,78 & 1,13 & 2,86 & 39,51 & 1,23 & 2,86 & 43,01 & M \\
\hline \multirow[t]{2}{*}{7} & 1,12 & 2,66 & 42,11 & 1,03 & 2,15 & 47,91 & 1,42 & 2,86 & 49,65 & 1,02 & 2,67 & 38,20 & M \\
\hline & 1,23 & 2,55 & 48,23 & 0,81 & 2,04 & 39,71 & 1,42 & 2,86 & 49,65 & 1,03 & 2,66 & 38,72 & M \\
\hline \multirow[t]{2}{*}{8} & 1,12 & 2,55 & 43,92 & 0,92 & 1,94 & 47,42 & 1,23 & 2,70 & 44,40 & 1,02 & 2,45 & 41,63 & M \\
\hline & 1,12 & 2,55 & 43,92 & 0,82 & 1,94 & 42,26 & 1,23 & 2,76 & 44,57 & 1,03 & 2,45 & 42,04 & M \\
\hline \multirow[t]{2}{*}{9} & 1,12 & 2,55 & 43,92 & 0,74 & 1,94 & 38,14 & 1,23 & 2,76 & 44,57 & 1,02 & 2,45 & 47,63 & M \\
\hline & 1,12 & 2,55 & 43,92 & 0,74 & 1,94 & 38,14 & 1,23 & 2,66 & 46,24 & 1,02 & 2,45 & 47,63 & M \\
\hline \multirow[t]{2}{*}{10} & 1,02 & 2,25 & 45,33 & 0,93 & 1,94 & 47,94 & 1,12 & 2,66 & 42,11 & 1,23 & 2,35 & 41,63 & M \\
\hline & 0,92 & 2,25 & 40,89 & 0,92 & 1,94 & 47,42 & 1,12 & 2,55 & 43,92 & 1,23 & 2,35 & 41,63 & M \\
\hline \multirow[t]{2}{*}{11} & 1,80 & 2,15 & 37,20 & 0,69 & 1,85 & 37,29 & 0,82 & 2,45 & 33,45 & 0,68 & 1,84 & 36,95 & SM \\
\hline & 0,76 & 2,04 & 37,25 & 0,69 & 1,85 & 37,29 & 0,82 & 2,45 & 33,45 & 0,68 & 1,84 & 36,95 & SM \\
\hline
\end{tabular}

* $\mathrm{CB}=$ braço curto; $\mathrm{T}=$ comprimento total; $\mathrm{IC}=$ índice centromérico; $\mathrm{SM}=$ submetacêntrico; $\mathrm{M}=$ metacêntrico. 

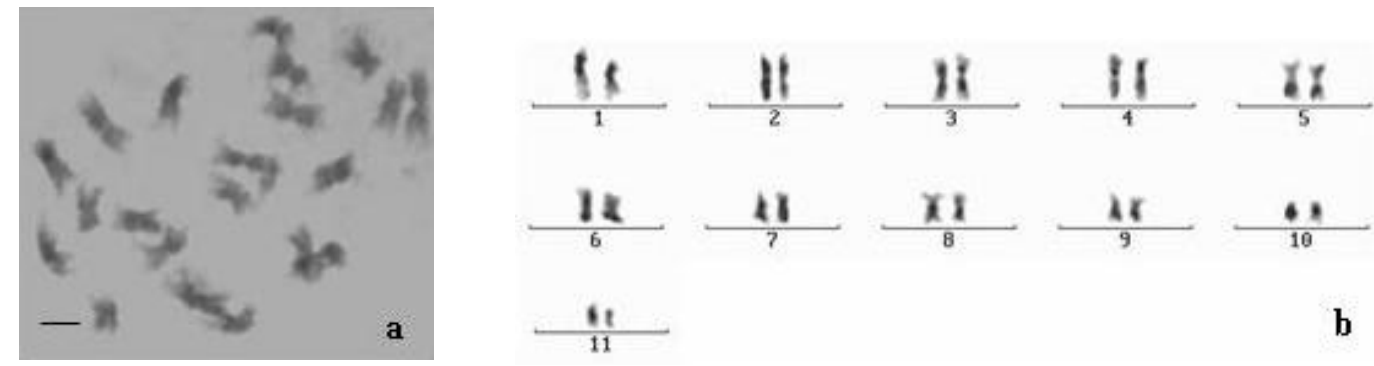

Figura 4. a. Metáfase mitótica de $S . \times$ buckleyi contendo flores de pigmentação rósea. b. Cariótipo mitótico $(2 \mathrm{n}=22$ cromossomos). Barra $=2,5 \mu \mathrm{m}$.

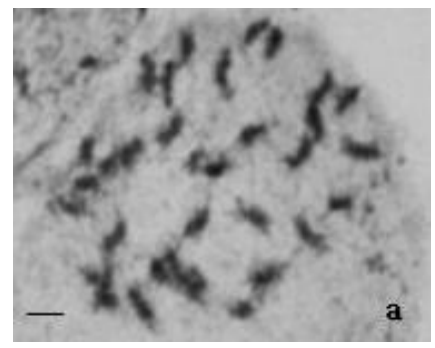

\section{1)}

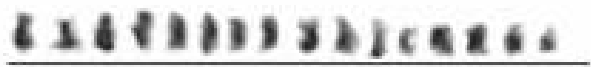

Figura 5. a. Metáfase mitótica de $S$. truncata contendo flores de pigmentação amarelada. b. Cromossomos mitóticos $(2 \mathrm{n}=34$ cromossomos $)$. Barra $=2,5 \mu \mathrm{m}$.

básico em Cactaceae é $\mathrm{n}=\mathrm{x}=11$ e os poliplóides são a principal variação (Stockwell 1935; Katagiri 1953; Remski 1954; Pinkava \& McLeod 1971; Weedin \& Powell 1978; Ross 1981). Katagiri (1953) encontrou, em Opuntiaea, níveis de ploidia de até 12x e Remski (1954) relatou a ocorrência de poliploidia em Mammillaria de até 24x o número básico haplóide. Pinkava \& McLeod (1971) comentaram que aneuploidias trissômicas e inversões estão presentes no gênero Opuntia. Segundo Weedin \& Powell (1978), alguns gêneros da família Cactaceae, como Opuntia, Echinocereus, Ferocactus e Ariocarpus possuem representantes poliplóides e aneuplóides. Stebbins (1971) apud Weedin \& Powell (1978) afirmou que a ocorrência de endomitoses, em plantas suculentas é comum. A planta caracterizada pela coloração amarelada das pétalas, pode ser um indivíduo triplóide trissômico $(2 \mathrm{n}=3 \mathrm{x}=33+1)$. Parks \& Boyle (2003) verificaram indivíduos diplóides $(2 \mathrm{n}=2 \mathrm{x}=22)$, triplóides $(2 \mathrm{n}=3 \mathrm{x}=33)$ e tetraplóides $(2 \mathrm{n}=4 \mathrm{x}=44)$, em clones cultivados de Schlumbergera. Outra hipótese a ser considerada é que esses indivíduos, com pétalas amareladas, podem ser híbridos advindos de um cruzamento entre $S$. truncata e uma espécie intragenérica com número cromossômico diferente, dando origem a um indivíduo fenotipicamente idêntico à $S$. truncata, mas com características citogenéticas distintas. Fischer (1962), apud Weedin \& Powell (1978), relatou uma provável ocorrência de híbridos dentro do gênero Opuntia. Segundo Benson (1969) apud Weedin \& Powell (1978), os cactos Opuntia schottii (Englem.) L. Benson e Opuntia stanlyi Englem. apresentam número cromossômico meiótico $\mathrm{n}=11$ e $\mathrm{n}=22$ cromossomos, respectivamente. Weedin \& Powell (1978) demonstraram que as espécies Opuntia atrispina Griffiths, Opuntia phaeacantha Englem. e Opuntia lindheimeri Englem. apresentam $2 \mathrm{n}=33$ cromossomos. No entanto, para o esclarecimento desses fatos, ainda são necessários estudos citogenéticos de meiose e de biologia molecular.

A técnica de bandeamento $\mathrm{C}$ permitiu a identificação de cada par cromossômico, tornando possível a montagem e o estudo do cariótipo para cada exemplar analisado. Os ideogramas (Fig. 6, 7 e 8) representam o padrão de banda-C para a espécie $S$. truncata com flores nas cores branca, vermelha e pink, respectivamente. A variedade amarelada não apresentou nenhum padrão de bandas-C. A Fig. 9 mostra padrão de bandeamento para o híbrido $S . \times$ buckleyi. Essa técnica, inicialmente descrita para vertebrados e invertebrados, especialmente para insetos, também mostra bons resultados quando 


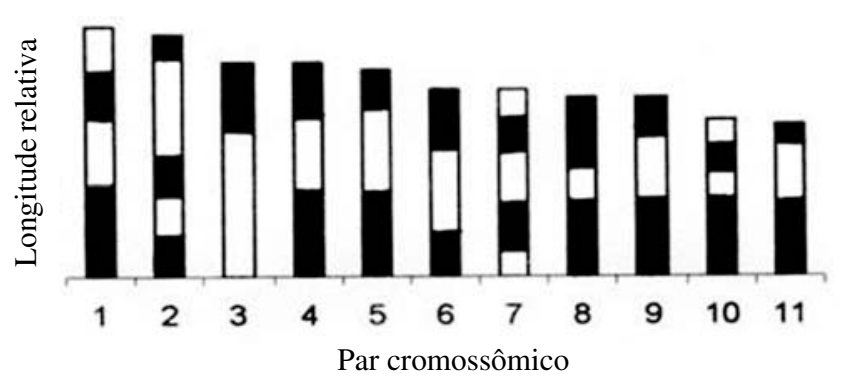

Figura 6. Ideograma de $S$. truncata, variedade com flores brancas, mostrando o padrão de Banda-C.

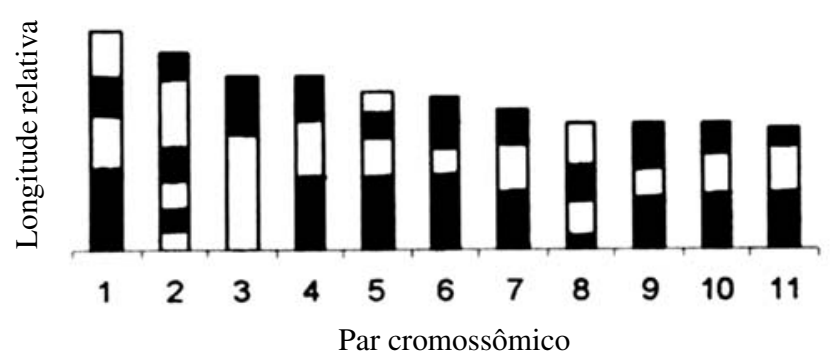

Figura 7. Ideograma de $S$. truncata, variedade com flores vermelhas, mostrando o padrão de Banda-C.

utilizada em vegetais. Ross (1981) advertiu que utilizando técnicas convencionais, geralmente, o reconhecimento de cromossomos específicos, em cactos, é difícil.

Nas Tab. 2 e 3 encontram-se os valores médios do comprimento total dos cromossomos das espécies em estudo, com seus respectivos desvios padrão. Essas medidas sugerem que o híbrido $S . \times$ buckleyi (rósea) e a espécie $S$. truncata, nas cores branca, vermelha e pink apresentam tamanhos cromossômicos semelhantes, mostrando-se, em média, maiores que 2,37 $\mu \mathrm{m}$. No entanto, diferem daqueles apresentados pelo exemplar de pétalas amareladas, que não ultrapassa 1,97 $\mu \mathrm{m}$. Stockwell (1935) demonstrou que em Opuntieae e em Cereeae, o comprimento cromossômico não excede $5 \mathrm{~m}$. Esse relato corrobora os dados encontrados no presente trabalho, pois o maior comprimento cromossômico encontrado, em média, não é superior a 3,58 m. Esses dados reforçam a teoria de ploidia, pois segundo Katagiri (1953) os cromossomos de cactáceas são, geralmente, pequenos e em plantas poliplóides esses cromossomos são ainda menores. Pesquisas relacionadas a estudos cariotípicos, dentro da família Cactaceae, não são descritos na literatura. Devido à ausência de detecção de centrômeros no exemplar S. truncata com pétalas amareladas, não foi possível obter o cariótipo e a classificação cromossômica para esta amostra.

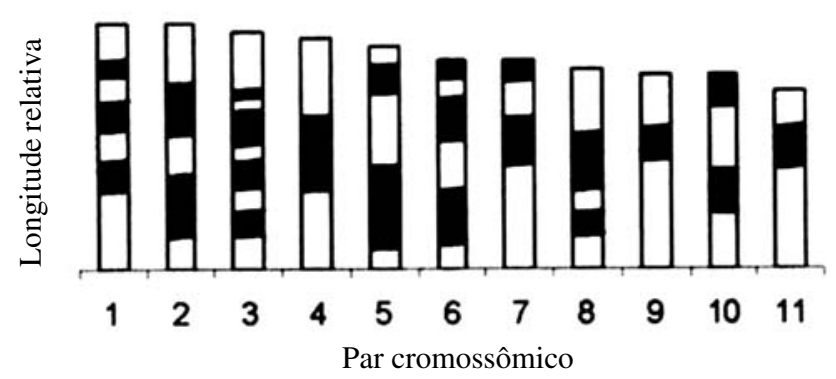

Figura 8. Ideograma de S. truncata, variedade com flores pink, mostrando o padrão de Banda-C.

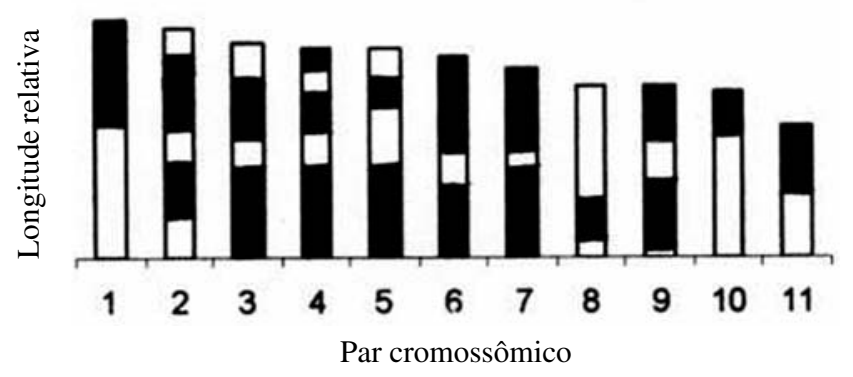

Figura 9. Ideograma de $S . \times$ buckleyi, variedade com flores róseas, mostrando o padrão de Banda-C.

A espécie $S$. truncata (branca, vermelha e pink) e o híbrido $S$. $\times$ buckleyi (rósea) apresentam raízes com índice de divisão mitótica aumentando nos meses de maio e junho. Após esse período, a quantidade de metáfases obtidas diminui significativamente. No entanto, a planta que possui flores com pigmentação amarelada nas pétalas $(S$. truncata) apresenta picos de obtenção de metáfases em dois períodos: maio - junho e setembro-outubro. Geralmente, os melhores períodos para a obtenção de metáfases coincidem com a época de florescimento (maio-junho) o que corresponde ao período outono-inverno (Figura 10).

Além do interesse teórico-científico, as análises citogenéticas presentes neste trabalho fornecem informações sobre o número cromossômico, posição centromérica e poliploidia dentro do grupo das cactáceas. A determinação do período anual em que ocorre maior divisão mitótica radicular, com conseqüente maior obtenção do número de metáfases, em Schlumbergera, facilita muito o trabalho dos citogeneticistas que pesquisam cactos pertencentes a este gênero. As análises cariotípicas convencionais podem atuar como ferramenta de auxílio na identificação de populações de $S$. truncata e $S$. x buckleyi possibilitando a produção de progênie híbrida para comercialização, sendo, portanto, de interesse aos citogeneticistas, melhoristas e taxonomistas. 
Tabela 2. Valores médios do comprimento total dos cromossomos da espécie S. truncata e do híbrido S. x buckleyi.

\begin{tabular}{|c|c|c|c|c|c|c|c|c|}
\hline \multirow[b]{2}{*}{ Par cromossômico } & \multicolumn{2}{|c|}{$\begin{array}{l}\text { S. truncata } \\
\text { branca }\end{array}$} & \multicolumn{2}{|c|}{$\begin{array}{l}\text { S. truncata } \\
\text { vermelha }\end{array}$} & \multicolumn{2}{|c|}{$\begin{array}{l}\text { S. truncata } \\
\text { pink }\end{array}$} & \multicolumn{2}{|c|}{$\begin{array}{l}S . \times \text { buckleyi } \\
\text { rósea }\end{array}$} \\
\hline & $\mathrm{MT}^{*}$ & $\delta$ & $\mathrm{MT}^{*}$ & $\delta$ & $\mathrm{MT}^{*}$ & $\delta$ & $\mathrm{MT}^{*}$ & $\delta$ \\
\hline \multirow[t]{2}{*}{1} & 3,58 & 0,2765 & 3,37 & 0,1155 & 3,37 & 0,2367 & 3,37 & 0,2899 \\
\hline & 3,58 & 0,2572 & 3,37 & 0,1000 & 3,37 & 0,5077 & 3,33 & 0,2899 \\
\hline \multirow[t]{2}{*}{2} & 3,47 & 0,3290 & 3,07 & 0,0635 & 3,37 & 0,3866 & 3,27 & 0,4313 \\
\hline & 3,47 & 0,2348 & 2,96 & 0,0635 & 3,37 & 0,3911 & 3,27 & 0,4313 \\
\hline \multirow[t]{2}{*}{3} & 3,07 & 0,1721 & 2,66 & 0,0513 & 3,27 & 0,3433 & 3,07 & 0,5020 \\
\hline & 3,07 & 0,1721 & 2,66 & 0,0577 & 3,23 & 0,3785 & 3,07 & 0,2828 \\
\hline \multirow[t]{2}{*}{4} & 3,07 & 0,2246 & 2,66 & 0,1582 & 3,17 & 0,3318 & 2,96 & 0,2121 \\
\hline & 3,07 & 0,1837 & 2,55 & 0,2138 & 3,17 & 0,2782 & 2,96 & 0,2121 \\
\hline \multirow[t]{2}{*}{5} & 2,96 & 0,1671 & 2,45 & 0,1582 & 3,07 & 0,3193 & 2,96 & 0,0707 \\
\hline & 2,96 & 0,2229 & 2,35 & 0,2138 & 3,07 & 0,3046 & 2,96 & 0,0707 \\
\hline \multirow[t]{2}{*}{6} & 2,66 & 0,1517 & 2,35 & 0,1582 & 2,86 & 0,3127 & 2,86 & 0,1414 \\
\hline & 2,66 & 0,1304 & 2,25 & 0,1000 & 2,86 & 0,3465 & 2,86 & 0,1414 \\
\hline \multirow[t]{2}{*}{7} & 2,66 & 0,8850 & 2,15 & 0,1250 & 2,86 & 0,3719 & 2,67 & 0,0707 \\
\hline & 2,55 & 0,1379 & 2,04 & 0,1732 & 2,86 & 0,3369 & 2,66 & 0,0707 \\
\hline \multirow[t]{2}{*}{8} & 2,55 & 0,3031 & 1,94 & 0,2082 & 2,77 & 0,3262 & 2,45 & 0,0990 \\
\hline & 2,55 & 0,1732 & 1,94 & 0,2050 & 2,76 & 0,2935 & 2,45 & 0,0707 \\
\hline \multirow[t]{2}{*}{9} & 2,55 & 0,1643 & 1,94 & 0,1212 & 2,76 & 0,2471 & 2,45 & 0,0000 \\
\hline & 2,55 & 0,1643 & 1,94 & 0,1212 & 2,66 & 0,2897 & 2,45 & 0,0707 \\
\hline \multirow[t]{2}{*}{10} & 2,25 & 0,1350 & 1,94 & 0,1790 & 2,66 & 0,3284 & 2,35 & 0,2192 \\
\hline & 2,25 & 0,2003 & 1,94 & 0,1000 & 2,55 & 0,3042 & 2,35 & 0,0778 \\
\hline \multirow[t]{2}{*}{11} & 2,15 & 0,1850 & 1,85 & 0,2367 & 2,45 & 0,3874 & 1,84 & 0,0707 \\
\hline & 2,04 & 0,2210 & 1,85 & 0,2367 & 2,45 & 0,4114 & 1,84 & 0,2899 \\
\hline $\begin{array}{l}\text { Tamanho médio dos } \\
\text { cromossomos }\end{array}$ & 2,81 & & 2,37 & & 2,92 & & 2,75 & \\
\hline Desvio-padrão médio & & 0,2314 & & 0,1436 & & 0,3379 & & 0,1870 \\
\hline
\end{tabular}

*MT $=$ Comprimento médio dos cromossomos de 15 metáfases $(\mu \mathrm{m}) ; \delta=$ desvio padrão.

Tabela 3. Valores médios do comprimento total dos cromossomos da espécie S. truncata com pétalas amareladas.

\begin{tabular}{|c|c|c|c|c|c|}
\hline \multirow[b]{2}{*}{ Par cromossômico } & \multirow[b]{2}{*}{$\mathrm{MT}^{*}$} & \multirow[b]{2}{*}{$\delta$} & $\begin{array}{c}\text { S. truncata } \\
\text { amarelada } \\
\end{array}$ & \multirow[b]{2}{*}{$\mathrm{MT}^{*}$} & \multirow[b]{2}{*}{$\delta$} \\
\hline & & & Par cromossômico & & \\
\hline \multirow[t]{2}{*}{1} & 2,96 & 0,2899 & 10 & 1,94 & 0,0778 \\
\hline & 2,66 & 0,4596 & & 1,94 & 0,0707 \\
\hline \multirow[t]{2}{*}{2} & 2,45 & 0,2121 & 11 & 1,94 & 0,0778 \\
\hline & 2,45 & 0,2192 & & 1,84 & 0,0071 \\
\hline \multirow[t]{2}{*}{3} & 2,35 & 0,2121 & 12 & 1,84 & 0,0071 \\
\hline & 2,35 & 0,2899 & & 1,84 & 0,0141 \\
\hline \multirow[t]{2}{*}{4} & 2,35 & 0,2192 & 13 & 1,64 & 0,1485 \\
\hline & 2,35 & 0,2899 & & 1,64 & 0,1485 \\
\hline \multirow[t]{2}{*}{5} & 2,15 & 0,1485 & 14 & 1,64 & 0,0707 \\
\hline & 2,15 & 0,2121 & & 1,64 & 0,0566 \\
\hline \multirow[t]{2}{*}{6} & 2,15 & 0,1414 & 15 & 1,64 & 0,0566 \\
\hline & 2,15 & 0,2192 & & 1,64 & 0,0707 \\
\hline \multirow[t]{2}{*}{7} & 2,05 & 0,2263 & 16 & 1,43 & 0,0566 \\
\hline & 2,04 & 0,2192 & & 1,43 & 0,0778 \\
\hline \multirow[t]{2}{*}{8} & 2,04 & 0,2899 & 17 & 1,23 & 0,0071 \\
\hline & 2,04 & 0,2121 & & 1,23 & 0,0010 \\
\hline \multirow[t]{4}{*}{9} & 1,94 & 0,0778 & & & \\
\hline & 1,94 & 0,0849 & & & \\
\hline & \multirow{2}{*}{\multicolumn{2}{|c|}{$\begin{array}{c}\text { Tamanho médio dos cromossomos } \\
\text { Desvio-padrão médio }\end{array}$}} & & 1,97 & \\
\hline & & & & & 0,1421 \\
\hline
\end{tabular}

*MT = Comprimento médio dos cromossomos de 15 metáfases $(\mu \mathrm{m}) ; \delta=$ desvio padrão. 


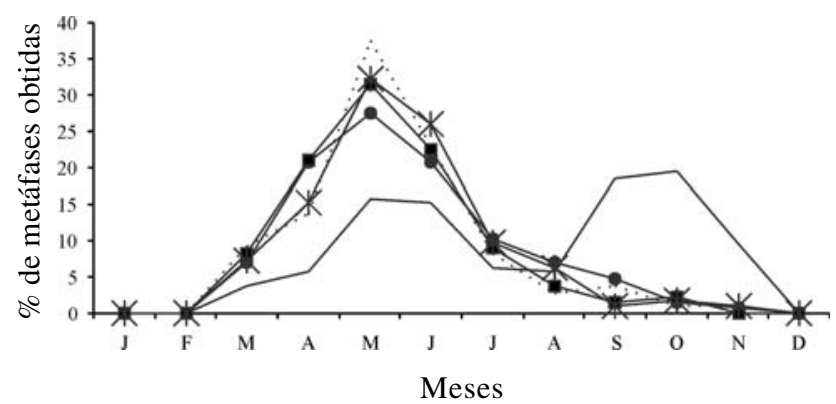

Figura 10. Determinação dos melhores períodos anuais para a obtenção de metáfases nas espécies $S$. truncata e $S . \times$ buckleyi. $(-\bullet-=$ Rósea $;-\bullet-=$ Pink $;-*-=$ Branca; $\cdots . .$. = Vermelha; $-=$ Amarelada).

\section{Referências bibliográficas}

Barthlott, W. \& Taylor, N.P. 1995. Notes towards a monograph of Rhipsalidaea (Cactaceae). Bradleya 13: 43-79.

Boyle, T.H. 1997. The Genetics of Self-incompatibility in the Genus Schlumbergera (Cactaceae). Journal of Heredity 88: $209-214$.

Boyle, T.H.; Karle, R. \& Han, S.S. 1995. Pollen Germination, Pollen Growth, Fruti Set, and Seed Development in Schlumbergera truncata and S. x buckleyi (Cactaceae). Journal American Society Horticulture Science 120(2): 313-317.

Guerra, M.S. \& Souza, M.J. 2002. Como observar cromossomos. Funpec Ed. Ribeirão Preto.
Katagiri. S. 1953. Chromosome numbers and polyploidy in certain Cactaceae. Cactus and Succulent Society of American 25: 141-142.

Levan, A.; Fredga, K. \& Sandberg. 1964. A Nomenclature for centromeric position on chromosomes. Hereditas 52(2): 201-220.

Martinez, A.P. 1976. Procedimento para facilitar el estudo de cromosomas en materials vegetales dificiles. Cuadernos G. Biological 5: 53-60.

O'Leary, M.C. \& Boyle, T.H. 1998. Segregation distortion at isozyme locus Lap-1 in Schlumbergera (Cactaceae) is caused by linkage with the gametophytic selfincompatibility (S) locus. The American Genetic Association 89: 206-210.

Parks, C. \& Boyle, T.H. 2003. Variation in ploidy level, fertility, and breeding behavior in cultivated Schlumbergera (Cactaceae). ISHS Acta Horticulturae 623: XXVI International Horticultural Congress: Plant genetic Resoucers.

Pinkava, D.J. \& McLeod, M.G. 1971. Chromosome numbers in some cacti of western North América. Brittonia 23: 171-176.

Remski, M.F. 1954. Cytological investigations in Mammillaria and some associated genera. Botanical Gazette 116: 163-171.

Ross, R. 1981. Chromosome Counts, Cytology, and Reproduction in the Cactaceae. American Journal of Botany 68(4): 463-470.

Stockwell P. 1935. Chromosome numbers of some the Cactaceae. Botanical Gazette 96: 565-570.

Weedin, J.F. \& Powell, A.M. 1978. Chromosome numbers in Chihuahuan Desert Cactaceae. Trans-Pecos Texas. American Journal of Botany 65(5): 531-537. 